Asia-Pacific Journal of Operational Research

Vol. 21, No. 4 (2004) 561-563

(C) World Scientific Publishing Co. \& Operational Research Society of Singapore

\title{
AUTHOR INDEX \\ Volume 21
}

Abbass, H. A., see Sarker, R.

$\mathbf{2}(2004) 225$

Ballestero, E., Selecting Textile Products by Manufacturing

Companies Under Uncertainty

$2(2004) 141$

Bardhan, A. K., see Kapur, P. K.

3 (2004) 333

Beaumont, N., Fitting a Table to a Page Using Nonlinear

Optimization

$\mathbf{2}(2004) 259$

3 (2004) 297

Bomze, I. M., see Churilov, L.

$\mathbf{2}(2004) 197$

Budhraja, R. K., see Kumar, N.

Burdett, R. L. \& Kozan, E., The Assignment of Individual

Renewable Resources in Scheduling

3 (2004) 355

Calvete, H. I. \& Galé, C., A Penalty Method for Solving Bilevel

Linear Fractional/Linear Programming Problems

$2(2004) 207$

Chandrasekhar, P., Natarajan, R. \& Yadavalli, V. S. S., A Study on a Two Unit Standby System with Erlangian Repair Time

Chang, C.-T., Inventory Models with Stock-Dependent Demand and Nonlinear Holding Costs for Deteriorating Items

Chang, S. Y., see Hwang, H.-C.

Chen, Z., Existence, Uniqueness, and Determinacy of a

Nonnegative Equilibrium Price Vector in Asset Markets with

General Utility Functions and an Elliptical Distribution

3 (2004) 393

Churilov, L., Bomze, I. M., Sniedovich, M. \& Ralph, D., Hyper

Sensitivity Analysis of Portfolio Optimization Problems

Craven, B. D., see Li, M. Q.

Curtis, N. J. \& Dortmans, P. J., A Dynamic Conceptual Model to Explore Technology-Based Perturbations to a Complex System:

The Land Force

Dortmans, P. J., see Curtis, N. J.

$4(2004) 463$

Du, J., see Jin, Z.

4 (2004) 463

3 (2004) 279

Galé, C., see Calvete, H. I.

2 (2004) 207

Ghazanfari, M. \& Nojavan, M., Reducing Inconsistency in Fuzzy AHP by Mathematical Programming Models

3 (2004) 379

Hong, Y., see Hwang, H.-C.

1 (2004) 117

2 (2004) 241

Huang, H. C., see Teng, S. Y. 
Hwang, H.-C., Chang, S. Y. \& Hong, Y., A Posterior

Competitiveness for List Scheduling Algorithm on Machines with Eligibility Constraints

1 (2004) 117

Hwang, S.-N., see Sueyoshi, T.

1 (2004) 69

Hwang, S.-N., see Sueyoshi, T.

2 (2004) 179

Jahanshahloo, G. R., Lotfi, F. H., Shoja, N. \& Tohidi, G.,

A Method for Generating All the Efficient Solutions of a 0-1

Multi-Objective Linear Programming Problem

$1(2004) 127$

Jha, P. C., see Kapur, P. K.

Jin, Z., Ohno, K. \& Du, J., An Efficient Approach for the

3 (2004) 333

Three-Dimensional Container Packing Problem with Practical

Constraints

3 (2004) 279

Kapur, P. K., Jha, P. C. \& Bardhan, A. K., Optimal Allocation of Testing Resource for a Modular Software

Kim, D. K., see Lim, J.-H.

Kim, W.-J., see Park, C.-K.

Kozan, E., see Burdett, R. L.

Kuan, S. N., Ong, H. L. \& Ng, K. M., Applying Metaheuristics to Feeder Bus Network Design Problem

Kumar, N., Budhraja, R. K. \& Mehra, A., Approximate Efficiency for $n$-set Multiobjective Fractional Programming

Lee, S., see Park, C.-K.

Li, M. Q. \& Craven, B. D., Some Properties of Optimal Multifunctions in Parametric Multiobjective Optimization with Set-Valued Maps

Lim, J.-H., Shin, S. W., Kim, D. K. \& Park, D. H., Bootstrap Confidence Intervals for Steady-State Availability

Lim, S. \& Park, S., A New Admissible Pivot Method for Linear Programming

3 (2004) 333

3 (2004) 407

1 (2004) 53

3 (2004) 355

$4(2004) 543$

2 (2004) 197

1 (2004) 53

Lin, C. \& Lin, Y., A Joint EOQ Model for Supplier and Retailer with Deteriorating Items

$1(2004) 1$

3 (2004) 407

$4(2004) 421$

$2(2004) 163$

Lin, C.-T. \& Wu, C.-R., Real Options: Batch Process and Market Entry/Exit Decisions Under Uncertainty

1 (2004) 35

Lin, C.-T., see Wu, C.-R.

Lin, Y., see Lin, C.

Liu, S. Q. \& Ong, H. L., Metaheuristics for the Mixed Shop Scheduling Problem

4 (2004) 499

$\mathbf{2}(2004) 163$

Lotfi, F. H., see Jahanshahloo, G. R.

Marín, Á. see Salmerón, J.

Mathirajan, M. \& Meenakshi, B., Experimental Analysis of Some

Variants of Vogel's Approximation Method

1 (2004) 97

1 (2004) 127

1 (2004) 9

Meenakshi, B., see Mathirajan, M.

$4(2004) 447$

4 (2004) 447

Mehra, A., see Kumar, N.

2 (2004) 197 
Natarajan, R., see Chandrasekhar, P.

Ng, K. M., see Kuan, S. N.

Nojavan, M., see Ghazanfari, M.

Ohno, K., see Jin, Z.

Ong, H. L., see Kuan, S. N.

Ong, H. L., see Liu, S. Q.

Ong, H. L., see Teng, S. Y.

Park, C.-K., Kim, W.-J., Lee, S. \& Park, S., Positive Sensitivity Analysis in Linear Programming

Park, D. H., see Lim, J.-H.

Park, S., see Lim, S.

Park, S., see Park, C.-K.

Ralph, D., see Churilov, L.

Ravi, V., Optimization of Complex System Reliability by Modified Great Deluge Algorithm

Salmerón, J. \& Marín, Á., A Convex Submodel with Application to System Design

Sarker, R. \& Abbass, H. A., Differential Evolution for Solving Multiobjective Optimization Problems

Shah, N. H., Probabilistic Order Level System when Items in Inventory Deteriorate and Delay in Payments is Permissible

Shin, S. W., see Lim, J.-H.

Shoja, N., see Jahanshahloo, G. R.

Sniedovich, M., see Churilov, L.

Sueyoshi, T. \& Hwang, S.-N., A Use of Nonparametric Tests for DEA-Discriminant Analysis: A Methodological Comparison

Sueyoshi, T. \& Hwang, S.-N., Parallel Network Computing Approach for DEA-RAM Measurement

Teng, S. Y., Ong, H. L. \& Huang, H. C., An Integer L-Shaped Algorithm for Time-Constrained Traveling Salesman Problem with Stochastic Travel and Service Times

Tohidi, G., see Jahanshahloo, G. R.

Wie, B.-W., Open-Loop and Closed-Loop Models of Dynamic Oligopoly in the Cruise Line Industry

Wu, C.-R. \& Lin, C.-T., The Choice of Foreign Production

Strategy and Timing of Decision Among Three Countries Under

Exchange Rate Uncertainty

Wu, C.-R., see Lin, C.-T.

Yadavalli, V. S. S., see Chandrasekhar, P.
3 (2004) 271

4 (2004) 543

3 (2004) 379

3 (2004) 279

4 (2004) 543

1 (2004) 97

2 (2004) 241

1 (2004) 53

3 (2004) 407

4 (2004) 421

1 (2004) 53

3 (2004) 297

4 (2004) 487

1 (2004) 9

2 (2004) 225

3 (2004) 319

3 (2004) 407

1 (2004) 127

3 (2004) 297

2 (2004) 179

1 (2004) 69

2 (2004) 241

1 (2004) 127

4 (2004) 517

4 (2004) 499

1 (2004) 35

3 (2004) 271 